\title{
Additive genetic variation in resistance of Nile tilapia (Oreochromis niloticus) to Streptococcus iniae and S. agalactiae capsular type Ib: Is genetic resistance correlated?
}

Craig A. Shoemaker ${ }^{a 1}$, Carlos A. Lozano ${ }^{b}$, Benjamin R. LaFrentz ${ }^{a}$, Julio C. García ${ }^{a}$, Esteban Soto ${ }^{\mathrm{c}}$, De-Hai Xu ${ }^{\mathrm{a}}$, Benjamin H. Beck ${ }^{\mathrm{a}}$ and Morten Rye $\mathrm{e}^{\mathrm{b1}}$

${ }^{a}$ United States Department of Agriculture-Agricultural Research Service (USDA-ARS), Aquatic Animal Health Research Unit, 990 Wire Road, Auburn, AL, 36832-4352, USA

${ }^{\mathrm{b}}$ Akvaforsk Genetics Center AS, Sjølsengvegen 22, N-6600 Sunndalsøra, Norway

${ }^{\mathrm{c}}$ University of California Davis School of Veterinary Medicine, Tupper Hall, Davis, CA 95616

${ }^{1}$ Corresponding authors: craig.shoemaker@ars.usda.gov and morten.rye@afgc.no 


\section{ABSTRACT}

Streptococcus iniae and S. agalactiae are both economically important Gram positive bacterial pathogens affecting the globally farmed tilapia (Oreochromis spp.). Historically, control of these bacteria in tilapia culture has included biosecurity, therapeutants and vaccination strategies. Genetic gains in performance traits have been realized for Nile tilapia (Oreochromis niloticus) and interest in breeding for disease resistance has recently received attention. The goal of this study was three fold: 1) to verify previous results demonstrating heritability of S. iniae resistance in Nile tilapia families using increased numbers of fish per family; 2) to determine if realized genetic gain in resistance and/or susceptibility to $S$. iniae can be obtained following positive assortative mating between parents with high or low estimated breeding values (EBV); and 3) to determine if resistance to S. iniae and S. agalactiae capsular type Ib is genetically correlated. A total of 144 and 130 full sib families were challenged intraperitoneally with S. iniae and intramuscularly with S. agalactiae Ib, respectively. Cumulative mortality at test end was $46 \%$ for S. iniae and $68 \%$ for S. agalactiae. There was a high additive genetic component found for survival in fish injected with $S$. iniae (estimated heritability $0.52 \pm 0.12$ ) and with S. agalactiae (estimated heritability $0.38 \pm 0.11$ ). The $S$. iniae challenge results confirmed additive genetic variation in resistance of Nile tilapia to $S$. iniae. We also demonstrated via assortative mating that genetic gain for survival to $S$. iniae is possible. The genetic correlation between resistance to $S$. iniae and $S$. agalactiae $\mathrm{Ib}$ was not significantly different from zero $\left(\mathrm{r}_{\mathrm{g}}=-0.30 \pm 0.19\right)$. The lack of correlation suggests if resistance to both Streptococcus sp. is desired, selection for both traits must be simultaneous. Selection of fish to improve survival to Streptococcus sp. may require a thorough understanding of the type of pathogen prevalent in the region so that custom genetic material may be tailored to meet the needs of the individual farm and/or region. Keywords: Streptococcus iniae; Streptococcus agalactiae Ib; selective breeding; Nile tilapia 


\section{Introduction}

Worldwide tilapia (Oreochromis spp.) sales were estimated at US \$7.6 billion in 2012 (FAO, 2014). Production is set to expand with estimates of production nearing 5,000,000 $\mathrm{t}$ (http://ag.arizona.edu/azaqua/ista/markets.htm, accessed March 4, 2016). Tilapia mortality due to climatic events and disease were recently suggested to be limiting growth of tilapia aquaculture (Thodesen et al. 2013). Streptococcus iniae (a non-groupable Streptococcus sp.; Pier and Madin, 1976) and S. agalactiae (group B Streptococcus; Vandamme et al. 1997) are economically significant Gram positive bacterial pathogens of tilapia (Eldar et al. 1994; Shoemaker et al. 2001; Agnew and Barnes 2007; Mian et al. 2009; Evans et al. 2015; Kayansamruaj et al. 2015; Soto et al. 2015; Delannoy et al. 2016) and have also been associated with potential for zoonotic infections (Weinstein et al. 1997; Liu et al. 2013; Delannoy et al. 2013). These reports highlight the negative impact of these emerging and/or reemerging diseases in farm raised tilapia. Tilapia are highly susceptible to S. agalactiae and capsular type Ib is virulent with lethal dose 50 values via intraperitoneal (ip) injection as few as 40 to 1000 colony forming units (cfu) fish ${ }^{-1}$ (Evans et al. 2015; Soto et al. 2015; Delannoy et al. 2016). Capsular type Ib isolates appear to be associated with clonal complex 552 that has not been reported from human cases of S. agalactiae (Delannoy et al. 2013; Rosinski-Chupin et al. 2013; Delannoy et al. 2016). Vaccination has been utilized to prevent $S$. iniae and S. agalactiae infection; however, the most effective method is via injection delivery (Eldar et al. 1997; Evans et al. 2004; Sheehan et al. 2009; Locke et al. 2010; Shoemaker et al. 2010; LaFrentz et al. 2011; Chen et al. 2012) that may not be cost effective. Other fish health management strategies such as a reduction of fish density (Shoemaker et al., 2000) and use of antibiotics (Gaunt et al., 2010) to control the 
infection have been practiced. Prevention and control of streptococcal disease in tilapia will require an integrated fish-health management strategy.

Selective breeding for growth and resistance to disease is a complimentary strategy for improving fish performance and health. LaFrentz et al. (2016) recently demonstrated that resistance to $S$. iniae is heritable (heritability estimate $=0.42 \pm 0.07$, observed binary scale) in Nile tilapia (O. niloticus). This is similar to reports in other fish species and pathogens illustrating that resistance to infectious disease can be moderately heritable (Silverstein et al., 2009; Leeds et al. 2010; Ødegård et al., 2011; Evenhuis et al., 2015). Research has illustrated the potential for genetic improvement of disease resistance in fish without adversely affecting performance (Bangera et al. 2011; Ødegård et al., 2011; Wiens et al., 2013). The goal of the study reported here was three fold: 1) to verify previous results demonstrating heritability of resistance to $S$. iniae in Nile tilapia using increased numbers of fish per family; 2) to determine if realized genetic gain in resistance and/or susceptibility to $S$. iniae can be obtained following positive assortative mating between parents with high or low estimated breeding values (EBV); and 3) to determine if resistance to $S$. iniae and $S$. agalactiae capsular type $\mathrm{Ib}$ is genetically correlated.

\section{Materials and methods}

\subsection{Fish, rearing conditions and transport}

Fish from the fourth generation (G4B1) of the Spring Genetics Nile tilapia breeding program were used for this study. Further details of the genetic material are provided in LaFrentz et al. (2016). A total of 144 full sib families were produced in June 2015 using 72 sires and 144 dams from the previous generation. Families were produced by natural mating in single 
pair breeding units over a period of $18 \mathrm{~d}$. A hierarchical nested design was used where each male was mated with two different females. Most of the families were produced using parents with the highest EBVs for growth (LaFrentz et al. 2016). However, 12 of the families were produced by positive assortative mating between parents within the 6 best and 6 worst families ranked according to their EBV for survival to S. iniae using the linear model (LaFrentz et al. 2016).

On average $61 \mathrm{~d}$ after egg collection fish from all families were pit-tagged and representatives from all families were communally stocked in two separate test units prior to transport from Spring Genetics' nucleus breeding center in Homestead, FL to the Aquatic Animal Health Research Unit (AAHRU) in Auburn, AL. Two groups of fish were transported. Sex was not recorded for the fish in the first group destined for S. iniae challenge (avg. weight, $29.5 \mathrm{~g}[\mathrm{sd}=14.9]$ ), since they were too young to record sex visually in a reliable way. Sex was recorded for all fish in the second group destined for S. agalactiae challenge (avg. weight, $179 \mathrm{~g}$ $[\mathrm{sd}=74.6])$.

\subsection{Fish rearing conditions at $A A H R U$}

\subsubsection{Group 1 fish for $S$. iniae challenge}

Upon arrival at the AAHRU, the tilapia for the $S$. iniae challenge were stocked into eight $340 \mathrm{~L}$ troughs for acclimation ( $\sim 356$ fish trough $\left.^{-1}\right)$ by placing transport water into the receiving tanks via pump. Afterwards, fish were netted into each tank and the local water supply was added to each tank $\left(\sim 0.5-1 \mathrm{~L} \mathrm{~min}^{-1}\right)$ to allow for a slow increase in water temperature $(26 \pm 2$ ${ }^{\circ} \mathrm{C}$ ) and adjustment to local water quality conditions. The fish were acclimated for a period of 20 d prior to bacterial challenge and fed daily (2-3\% body weight) with Aquamax Grower feed 
(PMI Nutrition International, Inc., Brentwood, MO, USA). The PIT tag number and date of death was recorded using an ISO handheld reader (ARE H5; AEG ID, Ulm, Germany) for mortalities that occurred during the acclimation period. The tilapia family information and PIT tag numbers were not provided to AAHRU in order for the experiment to be blinded. Water supply to the tanks was de-chlorinated city water at $26 \pm 2{ }^{\circ} \mathrm{C}$ with flow rates that allowed for the tank water to be exchanged $\sim 3$ times every $24 \mathrm{~h}$ for acclimation. Upon challenge tilapia were moved to a $5500 \mathrm{~L}$ tank receiving $\sim 13 \mathrm{~L} \mathrm{~min}^{-1}$ with water quality similar to LaFrentz et al. (2016). All procedures utilizing fish were approved by the USDA-ARS, AAHRU Institutional Animal Care and Use Committee.

\subsubsection{Group 2 fish for Streptococcus agalactiae challenge}

Following arrival at the AAHRU, the tilapia for the $S$. agalactiae challenge were acclimated for $13 \mathrm{~d}$ by stocking into sixteen $340 \mathrm{~L}$ troughs due to their larger average size $(\sim 170$ fish trough $\left.{ }^{-1}\right)$. All procedures were as described in the previous section.

\subsection{Streptococcus iniae growth conditions}

An archived stock of S. iniae generated following passage through tilapia was used for the $S$. iniae challenge (LaFrentz et al. 2016). Briefly, $50 \mu \mathrm{L}$ of thawed stock was inoculated into each of seven - $50 \mathrm{~mL}$ tubes containing tryptic soy broth (TSB; BD, Sparks, MD, USA) and then incubated at $28^{\circ} \mathrm{C}$ with shaking at 140 rotations per minute (rpm). After $24 \mathrm{~h}$ of growth, the tubes were aseptically combined into a single flask, the optical density (OD) measured at $540 \mathrm{~nm}$ was 1.3, and this culture was then diluted 1:5 for use in the bacterial challenge. The number of cfu $\mathrm{mL}^{-1}$ was determined by spread plating $100 \mu \mathrm{L}$ volumes of 10 -fold serial dilutions (in duplicate) onto sheep blood agar (SBA; Remel, Lenexa, KS, USA). Plates were incubated for 48 
$\mathrm{h}$ at $28^{\circ} \mathrm{C}$, and colonies were counted and averaged to enumerate the $\mathrm{cfu} \mathrm{mL}^{-1} \mathrm{using}$ standard procedures.

\subsection{Streptococcus iniae challenge}

The average weight of fish was $29.5 \mathrm{~g}(\mathrm{sd}=14.9 \mathrm{~g})$ determined $\sim 25 \mathrm{~d}$ prior to $S$. iniae challenge (i.e., at transport). All tilapia (groups of 20-50 fish) were anesthetized in 18 L buckets with $120 \mathrm{mg} \mathrm{L}^{-1}$ buffered tricaine methane sulfonate (MS-222; Argent Chemicals, Redmond, WA, USA) and injected ip with $100 \mu \mathrm{L}$ of 1:5 diluted $S$. iniae culture using a 26 gauge 3/8 inch needle. Each fish received $5.31 \times 10^{7} \mathrm{cfu}$, a dose that resulted in $\sim 50-70 \%$ mortality in preliminary experiments (data not shown). Prior to injection, the PIT tag number of each fish was recorded and following injection, fish were placed in clean water and transported to a 5,550 $\mathrm{L}$ tank to monitor recovery after handling and pathogen injection. Following challenge, fish were fed to satiation daily and dead fish were removed once or twice daily for $21 \mathrm{~d}$ and the PIT tag numbers and date of death were recorded. Re-isolation of $S$. iniae was attempted daily from a minimum of $20 \%$ of the dead fish by inoculating brain tissue onto SBA. Plates were incubated at $28{ }^{\circ} \mathrm{C}$ for $48 \mathrm{~h}$ and examined for the presence of beta-hemolytic colonies indicative of S. iniae. Upon termination of the challenge, all surviving fish were euthanized and PIT tag numbers were recorded.

\subsection{Streptococcus agalactiae Ib identification, growth conditions and passage through fish}

A virulent isolate of $S$. agalactiae LADL-05-108A was used for the bacterial challenge. This isolate was recovered from a diseased tilapia in Honduras that had been submitted to the Louisiana Animal Diagnostic Laboratory, Baton Rouge, LA (Soto et al. 2015). The capsular type of the isolate was confirmed based on the method of Imperi et al. (2010) with some 
modifications. Briefly, the method consisted of a multiplex PCR using the following primers cpsL F/R; cpsG F/R and cpsG-2-3-6 R; cpsJ 2-4 F, cps J-2 R; and cps J Ib F/R that allow identification of the most common capsular types associated with aquaculture. The capsular type was also confirmed serologically using antisera directed against capsular types Ia, Ib, II and III (Denka Seiken Co., Ltd., Japan; data not shown). Both methods confirmed the LADL-05-108A isolate as capsular type $\mathrm{Ib}$. The $S$. agalactiae isolate was grown for $24 \mathrm{~h}$ at $28{ }^{\circ} \mathrm{C}$ with shaking at $150 \mathrm{rpm}$ in TSB and used to pass the isolate through fish. Briefly, $100 \mu \mathrm{L}$ of the $24 \mathrm{~h}$ culture was injected into the peritoneal cavity (ip injection) of three to five tilapia (avg. weight, $48 \mathrm{~g}$ ). At $24 \mathrm{~h}$ post-injection, brain tissue from moribund fish were aseptically plated onto SBA and incubated at $28^{\circ} \mathrm{C}$ for $48 \mathrm{~h}$. A colony of $S$. agalactiae recovered from fish was passed from the SBA plate into TSB, incubated, and used to inject another group of tilapia as described above. This process was repeated one additional time for a total of three passages through tilapia. After the final passage, a colony was passed into TSB, grown, and then $20 \%$ glycerol stocks were prepared and frozen at $-80{ }^{\circ} \mathrm{C}$. The isolate was confirmed as $S$. agalactiae capsular type $\mathrm{Ib}$ by PCR as described above.

\subsection{Streptococcus agalactiae challenge}

The frozen stock of $S$. agalactiae following passage through tilapia was used for the challenge. Briefly, $100 \mu \mathrm{L}$ of thawed stock was inoculated into each of three $50 \mathrm{~mL}$ TSB broth tubes and then incubated at $28^{\circ} \mathrm{C}$ with shaking at $150 \mathrm{rpm}$. After $24 \mathrm{~h}$ of growth, each of the tubes were aseptically combined into a single flask and the OD measured at $540 \mathrm{~nm}$ was 0.88 . Seven replicate serial dilution series of the culture were prepared from 1:10 to 1:100000 in TSB and then the 1:100000 tubes were combined and placed on ice for use in injection of fish. The 
number of cfu $\mathrm{mL}^{-1}$ was determined by spread plating $100 \mu \mathrm{L}$ volumes of 10 -fold serial dilutions (in duplicate) onto SBA. Plates were incubated for $48 \mathrm{~h}$ at $28^{\circ} \mathrm{C}$, and colonies were counted and averaged to enumerate the cfu $\mathrm{mL}^{-1}$ using standard procedures.

Intramuscular (im) challenge was used based on pilot studies that indicated delivery of $1,998 \mathrm{cfu}^{\mathrm{cish}}{ }^{-1}$ resulted in $76 \%$ mortality at $21 \mathrm{~d}$ post challenge. Tilapia with an average weight at transport of $179.2 \mathrm{~g}(\mathrm{sd}=74.6)$ were anesthetized with $120 \mathrm{mg} \mathrm{L}^{-1}$ buffered tricaine methanesulfonate and im injected with $1,850 \mathrm{cfu}^{-1} \mathrm{sh}^{-1}$ using a 26 gauge $3 / 8$ inch needle. Briefly, groups of 20-50 fish were added to $18 \mathrm{~L}$ buckets containing anesthetic solution, anesthetized, removed with a net, pit tag recorded electronically, injected with $S$. agalactiae and then placed into a bucket containing clean water for transport to a single 5,500 L tank for monitoring of mortality post challenge. Fish were fed to satiation daily during challenge and dead fish were removed once or twice daily for $33 \mathrm{~d}$ and the PIT tag numbers and date of death were recorded. Re-isolation of S. agalactiae was completed on at least $20 \%$ of the fresh dead fish each day by culture of the brain on sheep blood agar. Plates were incubated at $28{ }^{\circ} \mathrm{C}$ for $48 \mathrm{~h}$ and then examined for bacterial colonies phenotypic of non-hemolytic S. agalactiae. A subsample of isolated bacteria was confirmed using capsular PCR typing as described above (data not shown). Upon termination of the challenge, all surviving fish were euthanized, examined for clinical signs of disease, and PIT tag numbers were recorded.

\subsection{Data analysis}

Survival at end of test (scored as 1 for fish alive and 0 for dead fish) was analyzed for both $S$. iniae and S. agalactiae. Fish from families with less than five fish were discarded from analysis. A total of 2681 records (representing 144 families) were used for S. iniae and 1900 
records for S. agalactiae (representing 130 families).

Univariate animal linear (1) and sire-dam threshold (2) models were used to obtain estimates of variance components of random effects for survival by the Restricted Maximum Likelihood (REML) using the ASReml software (Gilmour et al., 2009).

\subsubsection{Univariate linear animal model (Model 1)}

Model 1 (Univariate linear animal model): $\mathbf{y}=\mathbf{X b}+\mathbf{Z a}+\mathbf{W c}+\mathbf{e} ;$ where $\mathbf{y}$ is the vector of the phenotypic observations for survival; $\mathbf{X}$ is a design matrix for the fixed effects that links individual observations to the fixed effect class; $\mathbf{b}$ is the vector with the estimates of the fixed effects (sex only for S. agalactiae, production week, weight at start of challenge test as a covariate); $\mathbf{Z}$ is an incidence matrix that links the observations to the animal additive genetic effects, $\mathbf{a} \sim N\left(\mathbf{0}, \mathbf{A} \sigma_{a}^{2}\right)$ is a vector of random animal additive genetic effect, $\mathbf{W}$ is an incidence matrix that links the observations to the random environmental effects common to full-sibs, $\mathbf{c} \sim N\left(\mathbf{0}, \mathbf{I} \sigma_{c}^{2}\right)$ is a vector of random environmental effects common to full-sibs other than additive genetics; and $\mathbf{e}$ is a vector of random residuals. Finally, $\mathbf{A}$ is the numerator relationship matrix that describes the additive genetic relationship among all individuals included in a, and I is an identity matrix of appropriate size.

For Model 1 the significance of both the additive genetic effect and the effect common to full-sibs was assessed with the likelihood-ratio test (Lynch and Walsh, 1998):

$L R=-2\left(\log L_{R}-\log L_{F}\right)$, where $\log L_{R}$ is the $\log$ of the restricted likelihood of the reduced model (excluding each random effect at a time) and $\log \mathrm{L}_{\mathrm{F}}$ is the $\log$ of the restricted likelihood of the full model. The significance of LR was tested with a $\chi^{2}$ test with $\mathrm{df}=1$ (number of omitted variance components) and $\alpha=0.05$ for both random effects. For model 1, estimated 
heritability for survival was calculated as $h^{2}=\frac{\hat{\sigma}_{a}^{2}}{\hat{\sigma}_{a}^{2}+\hat{\sigma}_{c}^{2}+\hat{\sigma}_{e}^{2}}$, and the relative proportion of the environmental effects common to full-sibs as $c^{2}=\frac{\hat{\sigma}_{c}^{2}}{\hat{\sigma}_{a}^{2}+\hat{\sigma}_{c}^{2}+\hat{\sigma}_{e}^{2}}$.

\subsubsection{Univariate threshold sire-dam model (Model 2)}

Model 2 (Univariate threshold sire-dam model): $\boldsymbol{\lambda}=\mathbf{X b}+\mathbf{Z}_{\mathrm{S}} \mathbf{a}_{\mathrm{S}}+\mathbf{Z}_{\mathrm{D}} \mathbf{a}_{\mathbf{D}}+\mathbf{W c}+\mathbf{e}$; where

$\lambda_{\mathrm{i}}$ is the underlying liability which is assumed to be associated to the binary observation of animal $i\left(y_{i}\right)$ such that $\lambda_{\mathrm{i}} \leq 0$ gives $y_{i}=0$, and $\lambda_{\mathrm{i}}>0$ gives $y_{i}=1$. The vector $\lambda$ is a vector of all underlying liabilities. A Probit link function was used to relate the linear predictor scale to the observation scale for $S$. iniae. Due to problems with convergence, a Logit link function was used for $S$. agalactiae. The variance components were estimated on the underlying scale, where residual variance $\left(\sigma_{E}^{2}\right.$ ) was restricted to 1.0 , implying that $\mathbf{e} \sim N(\mathbf{0}, \mathbf{I}) ; \mathbf{X}, \mathbf{b}, \mathbf{W}, \mathbf{c}$ and $\mathbf{I}$ are the same as described in Model $1 ; \mathbf{Z}_{\mathbf{S}}$ and $\mathbf{Z}_{\mathbf{D}}$ are incidence matrices linking observations to the sire and dam additive genetic effects; $\mathbf{a}_{\mathbf{S}} \sim N\left(\mathbf{0}, \mathbf{A} \sigma_{S}^{2}\right)$ and $\mathbf{a}_{\mathbf{D}} \sim N\left(\mathbf{0}, \mathbf{A} \sigma_{D}^{2}\right)$ are vectors of the random sire and dam additive genetic effects, respectively; sire and dam variance are assumed to be equal $\sigma_{S}^{2}=\sigma_{D}^{2}=\sigma_{u}^{2}$. The effect common to full-sibs (c) included the environmental tank effects caused by separate rearing of the families until tagging and potential dominance and maternal effects. For each family, these effects are confounded, and thus fitted as a single random effect. Log-likelihood ratio tests cannot be used to test significance of random effects in the threshold models. For model 2, estimated heritability for survival was calculated as $h^{2}=\frac{4 \hat{\sigma}_{u}^{2}}{2 \hat{\sigma}_{u}^{2}+\hat{\sigma}_{c}^{2}+\hat{\sigma}_{e}^{2}}$, 
and the relative proportion of the environmental effects common to full-sibs as

$$
c^{2}=\frac{\hat{\sigma}_{c}^{2}}{2 \hat{\sigma}_{u}^{2}+\hat{\sigma}_{c}^{2}+\hat{\sigma}_{e}^{2}} .
$$

\subsubsection{Genetic correlation between survival to S. iniae and S. agalactiae}

The genetic correlation between survival to $S$. iniae and $S$. agalactiae was estimated using a bivariate model 1 (including sex effect only for $S$. agalactiae). The residual correlation was set to zero for the bivariate model 1 since only one trait was measured per fish. A reduced model where the additive genetic covariance between the two traits is assumed to be equal to zero was used to perform the likelihood-ratio test previously described and determine if the genetic correlation between both traits was significantly different from zero.

\section{Results}

\subsection{Fish acclimation and Streptococcus iniae challenge}

During transport and acclimation a total of 9 fish died. Accumulated mortality at the end of the challenge test was $46 \%$. Most of the fish died on day 1 and 2 after injection and by day 5 mortalities were less than $1 \%$ daily (Figure 1). The challenge was terminated at day 21 . Brain tissue samples were collected from 265 fish that died during the trial and $99 \%$ of these samples yielded pure cultures of S. iniae. There was high variation among mean family survival for fish injected ip with S. iniae, with survival ranging from 0 to $100 \%$, and a coefficient of variation of 55\% (Figure 2). The Pearson correlation between the mean family weight at shipment and $S$. iniae survival was negative but very low in magnitude and not statistically significant $(\mathrm{r}=-0.10$, $\mathrm{P}>0.05)$, suggesting that within the weight range for the families tested the initial weight of fish had little impact on survival. 


\subsection{Fish acclimation and Streptococcus agalactiae challenge}

During transport and acclimation a total of 68 fish died, and most acclimation mortalities occurred shortly after shipment (data not shown). Accumulated mortality at the end of the challenge test with S. agalactiae was $68 \%$. Mortalities began at $3 \mathrm{~d}$ post im injection, with the majority of the mortality occurring between days 5-11 after injection (Figure 1). After day 20 daily mortalities were less than $1 \%$. Mortality for S. agalactiae was higher than for S. iniae, but S. agalactiae mortality curve was less acute (Figure 1). Brain tissue samples were collected from 297 fish that died during the trial and 99\% of the fish samples collected yielded cultures of S. agalactiae. High variation among mean family survival for the fish injected with $S$. agalactiae (range from 0 to 100\%, CV 72\%) was observed (Figure 3). The Pearson correlation between the mean fish weight at shipment and S. agalactiae survival was close to zero $(\mathrm{r}=-0.04$, $\mathrm{P}>0.05$ ), suggesting that weight of fish at shipment did not influence survival.

\subsection{Additive genetic and environmental effect common to full-sibs}

There was a high additive genetic component found for survival in fish injected with either S. iniae or S. agalactiae (Table 2). The heritability estimate in the observed scale was 0.52 \pm 0.12 for $S$. iniae and $0.38 \pm 0.11$ for $S$. agalactiae. As expected, heritabilities were higher in the underlying liability scale, $0.74 \pm 0.16$ and $1.04 \pm 0.10$ for $S$. iniae and S. agalactiae, respectively. The additive genetic effect was significantly different from zero for both traits $(\mathrm{P}<$ 0.001; Log-likelihood ratio test Model 1). The environmental effect common to full-sibs was not significant for either trait ( $\mathrm{P}>0.05$; Log-likelihood ratio test Model 1), suggesting that separate rearing, maternal or epistatic effects did not affect the survival of the fish to the Streptococcus sp. tested. 


\subsection{Phenotypic and genetic correlation between survival to S. iniae and S. agalactiae}

The Pearson correlation between the phenotypic survival to S. iniae and S. agalactiae was negative but very low in magnitude and not statistically significant $(r=-0.07, P>0.05)$, suggesting there is no relationship between survival to S. iniae and S. agalactiae. The genetic correlation between S. iniae and S. agalactiae obtained with bivariate model 1 was not significantly different from zero $\left(\mathrm{r}_{\mathrm{g}}=-0.30 \pm 0.19, \mathrm{P}>0.05\right.$ in Log-likelihood ratio test $)$. When the number of full-sibs per family is high the correlation between the mid-parent EBV (using model 1) for S. iniae and S. agalactiae is close to the true genetic correlation between the traits. In this case this correlation was negative but not significant $(r=-0.08, P>0.05)$, confirming the lack of genetic relationship between survival to S. iniae and S. agalactiae.

\subsection{S. iniae parental groups}

Streptococcus iniae survival for the G4B1 families produced by assortative mating (see 2.1) is shown in Table 1. As expected based on the estimated heritability, offspring from families with the best $S$. iniae performance in 2014 had high survival in 2015 challenge test (average 88\%). Similarly, families with the worst parental performance had low survival in 2015 (10\%). The correlation between the mid-parent phenotypic S. iniae survival (based on data from 2014) and the offspring S. iniae survival (data from 2015) was positive and significant ( $\mathrm{r}=0.63$, $\mathrm{P}<0.001$ ). The regression of progeny survival on mid-parent offspring phenotypic survival was 0.77, which is a rough approximation of the realized heritability. In contrast, results suggest no relationship between the survival to the $S$. agalactiae in 2015 and the G4B1 families produced by assortative mating based on S. iniae index (Table 1). 


\section{Discussion}

The importance of tilapia to global aquaculture production has been increasing and led us to examine if selective breeding for resistance to Streptococcus spp. was possible. LaFrentz et al. (2016) pioneered the first disease resistance phenotyping of individual families in Nile tilapia against $S$. iniae and this report serves to validate and expand on the initial study. We have verified the first study results and confirmed the additive genetic variation in resistance of Nile tilapia to $S$. iniae using a larger number of fish per family (at least 14 fish represented for each family). The study reported here utilized smaller fish (29 g) as compared to larger sized fish (180 g) reported previously. The heritability estimate based on the observed binary scale was $0.52 \pm 0.12$, similar to $0.42 \pm 0.07$ reported by LaFrentz et al. (2016). Although injection of fish bypasses the innate immune response to some degree (i.e., skin and mucosal defenses), injection challenge has been utilized for assessing disease resistance to many bacterial fish pathogens (e.g., Leeds et al. 2010; Gjedrem and Gjøen 1995; Yáñez et al. 2013). In some cases, the genetic resistance observed following injection challenge has mirrored resistance in field trials (Gjøen et al. 1997; Wiens et al. 2013). Immersion or cohabitation challenges with S. iniae or S. agalactiae often result in low grade mortality and inconsistent results (Shoemaker et al. 2000; Soto et al. 2015). Our studies utilized injection challenge to ensure that each fish received the same pathogen dose and also used a large tank where all fish were maintained in the same environmental conditions throughout the challenge test.

Twelve tilapia families were produced via assortative mating in this study. Six families were produced based on high parental breeding values for survival to $S$. iniae and six families were produced based on low parental breeding values for survival to $S$. iniae (Table 1). Results demonstrated that mean survival of the offspring was $88 \%$ (range $60-100 \%$ ) for tilapia families 
produced using the high parental breeding values and only $10 \%$ (range $0-42 \%$ ) for families produced using poorer performing parents. These results clearly demonstrate that resistance to $S$. iniae is heritable and can be improved upon through selective breeding.

Streptococcus agalactiae has emerged as the predominant disease of tilapia (Mian et al. 2009; Chen et al. 2012; Delannoy et al. 2013; Evans et al. 2015). Due to the emergence of $S$. agalactiae, we hypothesized that disease resistance to $S$. iniae may be correlated with disease resistance to $S$. agalactiae due to the gross disease signs being similar (i.e., meningitis, cloudy eyes, pustule formation, exophthalmia, fecal casts, spiral swimming, etc.). In this regard, the same tilapia families were challenged with a $S$. agalactiae Ib isolate to examine for additive genetic variation in resistance to $S$. agalactiae. Results demonstrated substantial additive genetic variation for survival to $S$. agalactiae following im injection challenge with a heritability estimate of $0.38 \pm 0.11$ based on the observed binary scale derived from a linear animal model. Since cumulative end of test mortality was close to $50 \%$ in both traits, a simple cross sectional model such as Model 1 allows for accurate ranking of the families with respect to genetic merit (Ødegård et al. 2011).

For the S. agalactiae Ib challenge, we used im injection because pilot studies using ip injection were extremely lethal $\left(<100 \mathrm{cfu}^{\mathrm{fish}}{ }^{-1}\right.$ resulted in rapid and near $100 \%$ mortality, data not shown). Soto et al. (2015) and Delannoy et al. (2016) found similar results using ip injection challenge in gulf killifish (Fundulus grandis) and Nile tilapia, respectively. Our preliminary experiments showed a higher number of bacteria could be injected im that resulted in delayed mortality and reproducible results. Although, two different routes of challenge were used, results revealed no genetic correlation between resistance to $S$. iniae and S. agalactiae Ib.

No association was suggested when looking at the best and worst performing families for 
S. iniae survival (Table 1). Evenhuis et al. (2015) reported a small, favorable genetic correlation in resistance between two Flavobacterium spp. in rainbow trout (Oncorhynchus mykiss); however, our results suggest no association for $S$. iniae and $S$. agalactiae $\mathrm{Ib}$ in tilapia. Therefore, selection for disease resistance to $S$. iniae is not expected to indirectly affect resistance to $S$. agalactiae Ib. Bangera et al. (2011) reported high heritabilities and low or no genetic correlation among growth rate, resistance to Vibrio anguillarum and resistance to viral nervous necrosis in Atlantic cod (Gadus morhua L.) and suggested that genetic improvement for all traits was feasible. Based on our results in Nile tilapia, if resistance for both Streptococcus spp. is desired, selection must be for both traits simultaneously.

These results in part confirm the difficulty to control streptococcosis in commercial aquaculture. Research suggests that $S$. iniae and $S$. agalactiae are antigenically distinct as vaccination with a $S$. iniae bacterin did not provide protection against $S$. agalactiae Ia challenge (Evans et al. 2004). Furthermore, two main biotypes of $S$. agalactiae have been identified and it has been demonstrated that immunity to S. agalactiae is biotype specific (Sheehan et al. 2009). In this study, the challenge isolate used was a non-hemolytic S. agalactiae Ib (similar to biotype 2 described by Sheehan et al. 2009) that appears to be the predominant type in Asia (China, Indonesia, Vietnam and Philippines) and Central, North and South America (Soto et al. 2015), and has recently been suggested to be fish associated and/or fish specific (Rosinski-Chupin et al. 2013; Delannoy et al. 2016). Future testing may include the other predominant capsular types (Ia and III; biotype 1) that are more prevalent in S. agalactiae infected tilapia in the Middle East, Thailand, Malaysia and Singapore (Evans et al. 2002; Sheehan et al. 2009; Liu et al. 2013; Kayansamruaj et al. 2015; Delannoy et al. 2016). Selection of fish to improve survival to Streptococcus sp. may require a thorough understanding of the type of pathogen prevalent in the 
region so that custom genetic material may be tailored to meet the needs of the individual farm and/or region.

\section{Acknowledgments}

Funding and Nile tilapia families for this research was provided under a Material Transfer Agreement - Cooperative Research and Development Agreement (MTA-CRADA No. 58-6010-6-005) between Spring Genetics and United States Department of Agriculture Agricultural Research Service. The authors acknowledge the excellent technical assistance of José Ospina and Hideyoshi Segovia Uno (Spring Genetics) and Paige Mumma, Curtis Day, Ning Qin (USDA-ARS). This research was also supported by USDA-ARS CRIS Project No. 601032000-026-00D, Pathogen characterization, host immune response and development of strategies to reduce losses to disease in aquaculture. Mention of trade names or commercial products in this publication is solely for the purpose of providing specific information and does not imply recommendation or endorsement by the United States Department of Agriculture. 


\section{References}

Agnew, W., Barnes, A.C. 2007. Streptococcus iniae: An aquatic pathogen of global veterinary significance and a challenging candidate for reliable vaccination. Vet. Microbiol. 122, 115.

Bangera, R., Ødegård, J., Praebel, K.A., Mortensen, A., Nielsen, H.M. 2011. Genetic correlations between growth rate and resistance to vibriosis and viral nervous necrosis in Atlantic cod (Gadus morhua L.). Aquaculture 317, 67-73.

Chen, M., Wang, R., Li, L-P., Liang, W-W., Li, J., Huang, Y., Lei, A-Y, Huang, W-Y., Gan, X. 2012. Screening vaccine candidate strains against Streptococcus agalactiae of tilapia based on PFGE genotype. Vaccine 30, 6088-6092.

Delannoy, C., Crumlish, M., Fontaine, M.C., Pollock, J., Foster, G., Dagleish, M.P., Turnbull, J.F., Zadoks, R.N. 2013. Human Streptococcus agalactiae strains in aquatic mammals and fish. BMC Microbiol., 13:41.

Delannoy, C., Zadoks, R.N., Crumlish, M., Rodgers D., Lainson, F.A., Ferguson, H.W., Turnbull, J., Fontaine, M.C. 2016. Genomic comparison of virulent and non-virulent Streptococcus agalactiae in fish. J. Fish Dis. 39, 13-29.

Eldar, A., Bejerano, Y., Bercovier, H. 1994. Streptococcus shiloi and Streptococcus difficile: two new streptococcal species causing meningoencephalitis in fish. Current Microbiology 28, $139-143$.

Eldar, A., Horovitcz, A., Bercovier, H. 1997. Development and efficacy of a vaccine against Streptococcus iniae infection in farmed rainbow trout. Vet. Immunol. Immunopathol. 56, $175-183$. 
Evans, J.J., Klesius, P.H., Glibert, P.M., Shoemaker,C.A., AlSarawi, M.A., Landsberg, J., Duremdez, R., Marzouk, A. Al. and AlZenki, S. 2002. Characterization of betahemolytic Group B Streptococcus agalactiae in cultured sea bream, Sparus auratus 1. and wild mullet, Liza klunzingeri (Day), in Kuwait. J. Fish Dis. 25, 505-513.

Evans, J.J., Shoemaker, C.A., Klesius, P.H. 2004. Efficacy of Streptococcus agalactiae (Group B) vaccine in tilapia (Oreochromis niloticus) by intraperitoneal and bath immersion administration. Vaccine 22, 3769-3773.

Evans, J.J., Pasnik, D.J., Klesius, P.H. 2015. Differential pathogenicity of five Streptococcus agalactiae isolates of diverse geographic origin in Nile tilapia (Oreochromis niloticus L.) Aquac. Res. 46, 2374-2381.

Evenhuis, J.P., Leeds, T.D., Marancik, D.P., LaPatra, S.E., Wiens, G.D. 2015. Rainbow trout (Oncorhynchus mykiss) resistance to columnaris disease is heritable and favorably correlated with bacterial cold water disease resistance. J. Anim. Sci. 93, 1546-1554.

FAO, 2014. Fishery and Aquaculture Statistics 2012, Rome, Italy, pp. 105.

Gaunt, P.S., Endris, R., McGinnis, A., Baumgartner, W., Camus, A., Steadman, J., Sweeney, D., Sun, F. 2010. Determination of florfenicol dose rate in feed for control of mortality in Nile tilapia infected with Streptococcus iniae. J. Aquat. Anim. Health 22, 158-166.

Gilmour, A.R., Gogel, B.J., Cullis, B.R., Welham, S.J., Thompson, R. 2009. ASReml User Guide, Release 3.0. VSN, Hemel Hempstead, UK.

Gjedrem, T., Gjøen, H.M. 1995. Genetic variation in susceptibility of Atlantic salmon, Salmo salar L., to furunculosis, BKD and cold water vibriosis. Aquaculture Res. 26, 129-134.

Gjøen, H.M., Refstie, T., Ulla, O., Gjerde, B. 1997. Genetic correlations between survival of Atlantic salmon in challenge and fieled tests. Aquaculture 158, 277-288. 
Imperi M., Pataracchia M., Alfarone G., Baldassarri L., Orefici G., Creti R. (2010) A multiplex PCR assay for the direct identification of the capsular type (Ia to IX) of Streptococcus agalactiae. J. Microbiol. Methods 80, 212-214.

Kayansamruaj, P., Pirarat, N., Kondo, H., Hirono, I., Rodkhum, C. 2015. Genomic comparison between pathogenic Streptococcus agalactiae isolated from Nile tilapia in Thailand and fish-derived ST7 strains. Infection, Genetics and Evolution 36, 307-314.

LaFrentz, B.R., Shoemaker, C.A., Klesius, P.H. 2011. Immunoproteomic analysis of the antibody response obtained in Nile tilapia following vaccination with a Streptococcus iniae vaccine. Vet. Microbiol. 152, 346-352.

LaFrentz, B.R., Lozano, C.A., Shoemaker, C.A., García, J.C., Xu, D-H, Løvoll. M., Rye, M. 2016. Controlled challenge experiment demonstrates substantial additive genetic variation in resistance of Nile tilapia (Oreochromis niloticus) to Streptococcus iniae. Aquaculture. In Press.

Leeds, T.D., Silverstein, J.T., Weber, G.M., Vallejo, R.L., Palti, Y., Rexroad III, C.E., Evenhuis, J., Hadidi, S., Welch, T.J., Wiens, G.D. 2010. Response to selection for bacterial cold water disease resistance in rainbow trout. J. Anim. Sci. 88, 1936-1946.

Liu, G., Zhang, W., Lu, C. 2013. Comparative genomics analysis of Streptococcus agalactiae reveals that isolates from cultured tilapia in China are closely related to the human strain A909. BMC Genomics 17, 775.

Locke, J.B., Vicknair, M.R., Ostland, V.E., Nizet, V., Buchanan, J.T. 2010. Evaluation of Streptococcus iniae killed bacterin and live attenuated vaccines in hybrid striped bass through injection and bath immersion. Dis. Aquat. Org. 89, 117-123. 
Lynch, M., Walsh, B., 1998. Genetics and Analysis of Quantitative Traits. Sinauer Associates., Inc., Massachusetts. USA (980 pp.).

Mian, G.F., Godoy, D.T., Leal, C.A.G., Yuhara, T.Y., Costa, G.M., Figueiredo, H.C.P. 2009. Aspects of the natural history and virulence of $S$. agalactiae infection in Nile tilapia. Vet. Micro. 136, 180-183.

Ødegård, J., Baranski, M., Gjerde, B., Gjedrem, T. 2011. Methodology for genetic evaluation of disease resistance in aquaculture species: Challenges and future prospects. Aquaculture Res. 42, 103-114.

Pier, G.B., Madin, S.H. 1976. Streptococcus iniae sp. nov., a beta hemolytic streptococcus isolated from an Amazon freshwater dolphin, Inia geoffrensis. Int. J. Syst. Bacteriol. 26, $545-553$.

Rosinski-Chupin, I., Sauvage, E., Mairey, B., Mangenot, S., Ma, L., DaCunha, V., Rusniok, C., Bouchier, C., Barbe, V., Glaser, P. 2013. Reductive evolution in Streptococcus agalactiae and emergence of a host adapted lineage. BMC Genomics 14, 252.

Sheehan, B., Labrie, L., Lee, Y.S., Wong, F.S., Chan, J., Komar, C., Wendover, N., Grisez, L. 2009. Streptococcosis in tilapia; Vacccination effective against main strep species. Global Aquaculture Advocate July/August 72-74.

Shoemaker, C.A., Evans, J.J., Klesius, P.H. 2000. Density and dose: factors affecting mortality of Streptococcus iniae infected tilapia (Oreochromis niloticus). Aquaculture 188, 229235.

Shoemaker, C.A., Klesius, P.H., Evans, J.J. 2001. Prevalence of Streptococcus iniae in tilapia, hybrid striped bass, and channel catfish on commercial fish farms in the United States. Am. J. Vet. Res. 62, 174-177. 
Shoemaker, C.A., LaFrentz, B.R., Klesius, P.H., Evans, J.J. 2010. Protection against heterologous Streptococcus iniae isolates using a modified bacterin vaccine in Nile tilapia, Oreochromis niloticus (L.). J. Fish Dis. 33, 537-544.

Silverstein, J.T., Vallejo, R.L., Palti, Y., Leeds, T.D., Rexroad III, C.E., Welch, T.J., Wiens, G.D., Ducrocq, V. 2009. Rainbow trout resistance to bacterial cold-water disease is moderately heritable and is not adversely correlated with growth. J. Anim. Sci. 87, 860867.

Soto, E., Wang, R., Wiles, J., Baumgartner, W., Green, C., Plumb, J., Hawke, J. 2015. Characterization of isolates of Streptococcus agalactiae from diseased farmed and wild marine fish from the U.S. Gulf Coast, Latin America and Thailand. J. Aquat. Anim. Health 27, 123-134.

Thodesen, J., Rye, M., Wang, Y.-X., Li, S.-J., Bentsen, H.B., Gjedrem, T. 2013. Genetic improvement of tilapias in China: Genetic parameters and selection responses in growth, pond survival and cold-water tolerance of blue tilapia (Oreochromis aureus) after four generations of multi-trait selection. Aquaculture 396-399, 32-42.

Vandamme, P., Devriese, L.A., Pot, B., Kersters, Kl, and Melin, P. 1997. Streptococcus difficile is a nonhemolytic Group B, type Ib Streptococcus. Inter. J. Syst. Bacteriol. 47: 81-85.

Weinstein, M.R., Litt, M., Kertesz, D.A., et al. 1997. Invasive infections due to a fish pathogen, Streptococcus iniae. N. Engl. J. Med. 337, 589-594.

Wiens, G.D., LaPatra, S.E., Welch, T.J., Evenhuis, J.P., Rexroad III, C.E., Leeds, T.D. 2013. Onfarm performance of rainbow trout (Oncorhynchus mykiss) selectively bred for resistance to bacterial cold water disease: Effect of rearing environment on survival phenotype. Aquaculture 388-391, 128-136. 
Yáñez, J.M., Bangera, R., Lhorente, J.P., Oyarzún, M., Neira, R. 2013. Quantitative genetic variation of resistance against Piscirickettsia salmonis in Atlantic salmon (Salmo salar). Aquaculture 414-415, 155-159. 


\section{Table 1}

Survival results for the families produced with assorative mating for high and low resistance to Streptococcus iniae.

\begin{tabular}{|c|c|c|c|c|c|c|c|c|}
\hline \multirow{2}{*}{$\begin{array}{l}\text { Assortative } \\
\text { mating } \\
\text { groups }\end{array}$} & \multirow{2}{*}{$\begin{array}{l}\text { Number } \\
\text { of } \\
\text { families }\end{array}$} & \multirow{2}{*}{$\begin{array}{l}\text { Avg_S. } \\
\text { iniae index } \\
\text { parents }\end{array}$} & \multicolumn{3}{|c|}{$\begin{array}{l}\text { Percentage survival to } S \text {. } \\
\text { iniae in } 2015\end{array}$} & \multicolumn{3}{|c|}{$\begin{array}{l}\text { Percentage survival to } S \text {. } \\
\text { agalactiae in } 2015\end{array}$} \\
\hline & & & Average & Min & Max & Average ${ }^{* * * *}$ & Min & Max \\
\hline none & 132 & 101.6 & 54 & 0 & 100 & 31 & 0 & 89 \\
\hline yes_high & 6 & 121.9 & 88 & 60 & 100 & 19 & 7 & 50 \\
\hline yes_low & 6 & 80.2 & 10 & 0 & 42 & 40 & 18 & 59 \\
\hline Total & 144 & & & & & & & \\
\hline \multicolumn{9}{|c|}{ *Index $($ mean $=100, s d=10) . A v g=($ sire index + dam index $) / 2$} \\
\hline
\end{tabular}




\section{Table 2}

Survival variance components, estimated heritability $\left(\mathrm{h}^{2} \pm \mathrm{se}\right)$ and effect common to full-sibs $\left(\mathrm{c}^{2}\right.$ \pm se) for the univariate linear animal model (Model 1) and the univariate threshold sire-dam model (Model 2) for traits (Streptococcus iniae and S. agalactiae).

\begin{tabular}{|c|c|c|c|c|}
\hline \multirow[b]{2}{*}{ Parameter } & \multicolumn{2}{|c|}{ Model 1 (observed scale) } & \multicolumn{2}{|c|}{ Model 2 (underlying scale) } \\
\hline & S. iniae & S. agalactiae & S. iniae ${ }^{*}$ & S. agalactiae** \\
\hline$\sigma^{2}$ sire & - & - & 0.326 & 0.544 \\
\hline$\sigma^{2}$ dam & - & - & 0.326 & 0.544 \\
\hline$\sigma^{2}$ additive & 0.126 & 0.083 & 1.305 & 2.175 \\
\hline$\sigma^{2}$ common env. & 0.009 & 0.001 & 0.000 & 0.257 \\
\hline$\sigma^{2} \mathrm{e}$ & 0.109 & 0.136 & 1.000 & 1.000 \\
\hline$h^{2} \pm s e$ & $0.52 \pm 0.12$ & $0.38 \pm 0.11$ & $0.74 \pm 0.16$ & $1.04 \pm 0.10$ \\
\hline$c^{2} \pm s e$ & $0.04 \pm 0.04$ & $0.00 \pm 0.04$ & $0.03 \pm 0.05$ & $0.00 \pm 0.00$ \\
\hline
\end{tabular}

* PROBIT link function

** LOGIT link function 
Fig. 1. Cumulative percent mortality in Nile tilapia following challenge with Streptococcus iniae by intraperitoneal injection $(\bullet)$ and $S$. agalactiae Ib challenge by intramuscular injection ( $(\mathbf{})$.

Fig. 2. The number of fish per family (line; right y-axis) and mean survival (bars; left y-axis) of 144 Nile tilapia families following challenge with Streptococcus iniae by intraperitoneal injection. Families with less than five fish at the onset of the bacterial challenge were excluded from the data analysis (see section 2.7. Data Analysis).

Fig. 3. The number of fish per family (line; right y-axis) and mean survival (bars; left y-axis) of 130 Nile tilapia families following challenge with Streptococcus agalactiae by intramuscular injection. Families with less than five fish at the onset of the bacterial challenge were excluded from the data analysis (see section 2.7. Data Analysis). 


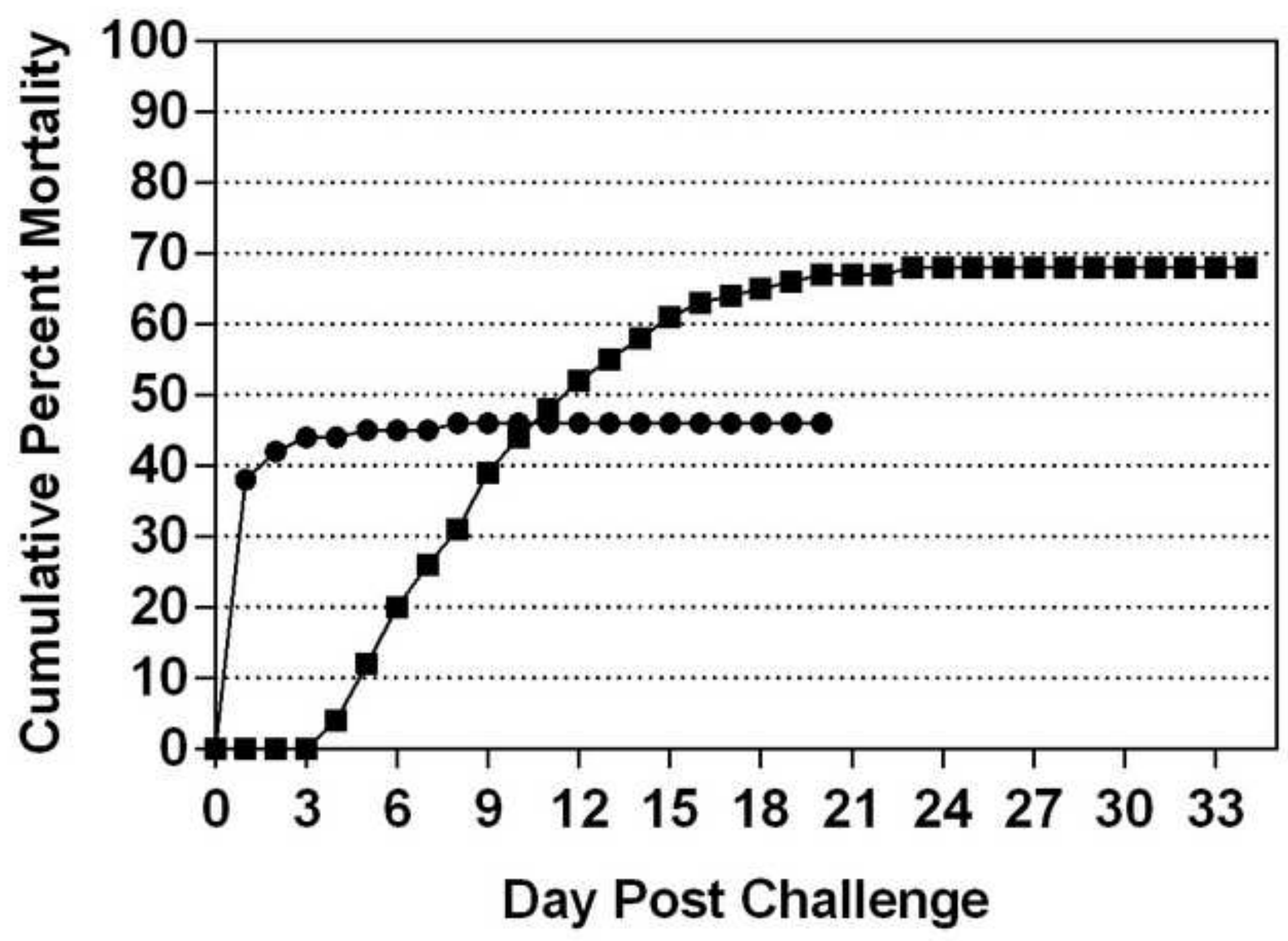




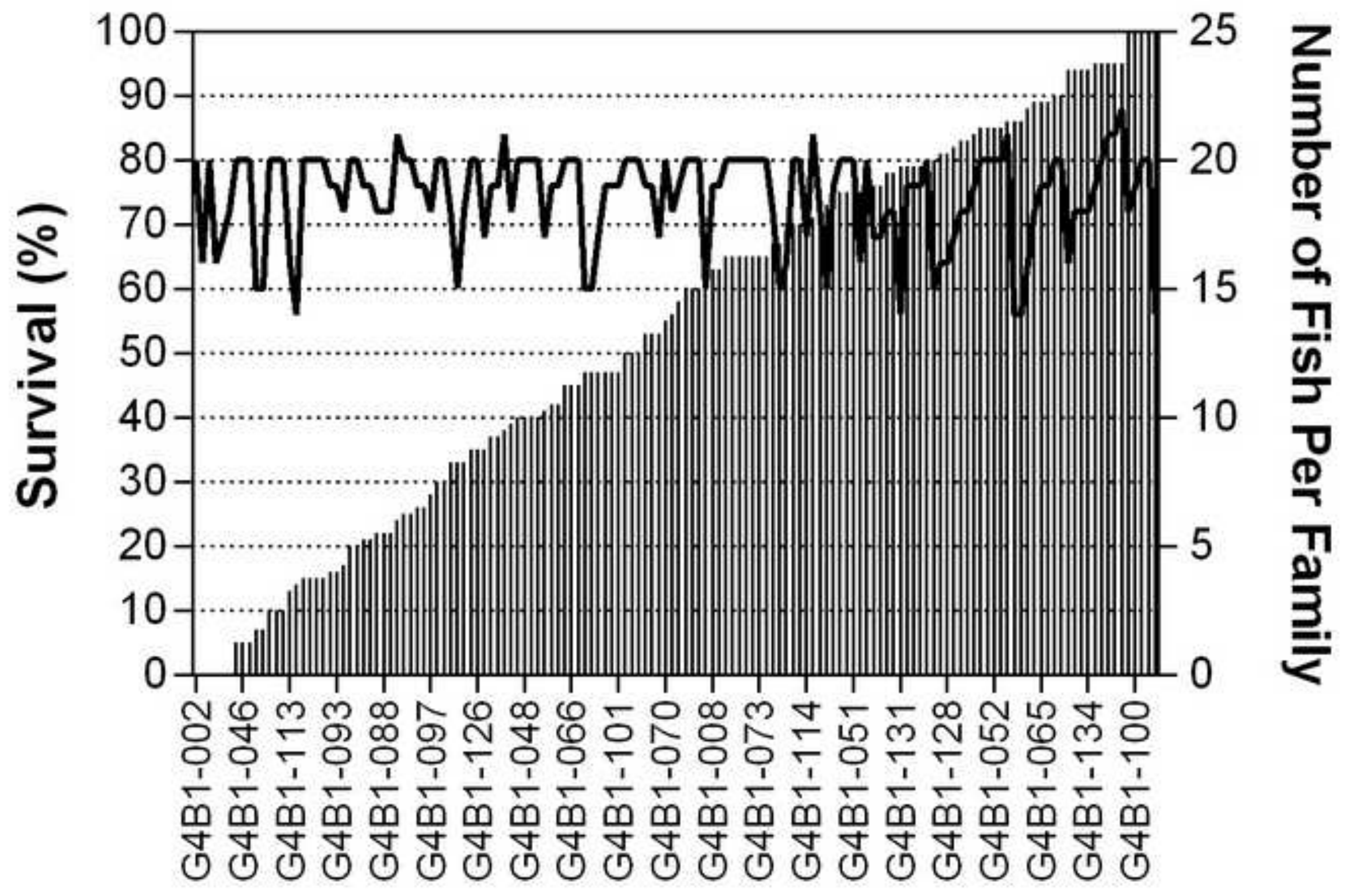

Families 


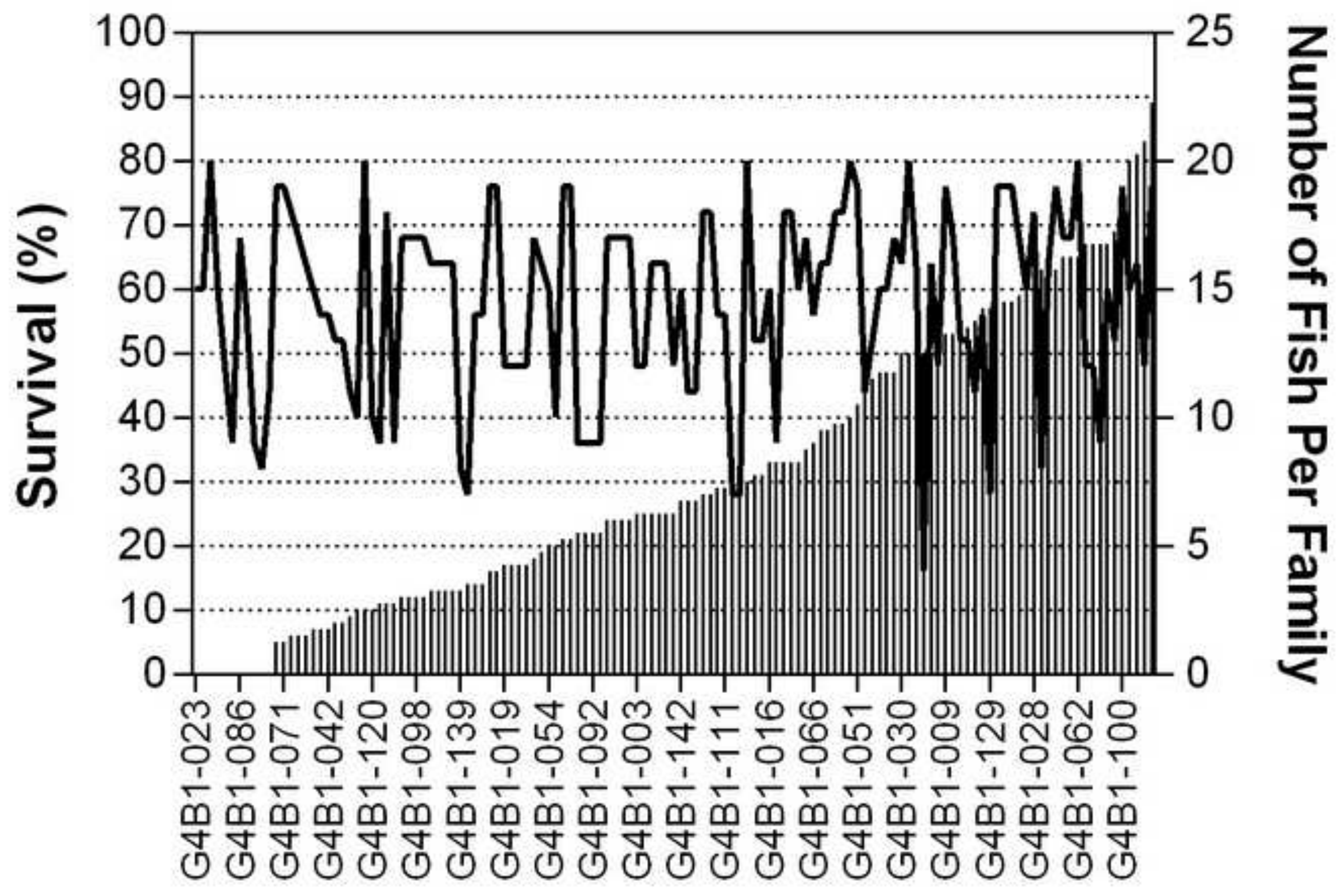

Families 